\title{
Number of Paths and Neighbours Effect on Multipath Routing in Mobile Ad Hoc Networks
}

\author{
Oday Jerew \\ School of Information Systems and Accounting \\ University of Canberra \\ Canberra ACT 2617, Australia \\ oday.jerew@canberra.edu.au
}

\author{
Kim Blackmore \\ The Australian National University \\ Canberra ACT 0200, Australia \\ Kim.Blackmore@anu.edu.au
}

\author{
Masoud Mohammadian \\ School of Information Systems and Accounting \\ University of Canberra \\ Canberra ACT 2617, Australia \\ masoud.mohammadian@canberra.edu.au
}

\begin{abstract}
In this paper the number of paths in a multipath routing protocol and distribution of neighbour nodes in the discovered routes are studied for networks with frequent topology changes. Specifically, we consider how the placement of neighbour nodes, which are used by the source node to reach a destination in a multipath routing protocol, affects total network power consumption. We develop an analytical model, verified by simulation, which shows that, while the network performance is affected by source node speed and route length, it also depends on the geometric arrangement of neighbour nodes around the source node. The results reveal that total network power consumption is minimized when the moving source node has six well distributed neighbour nodes, and one path to the destination via each neighbour node.
\end{abstract}

\section{INTRODUCTION}

In mobile ad hoc networks, nodes move freely in arbitrary directions and speeds that lead to frequent links failure and new routing paths need to be discovered. The cached routes become outdated, and hence invalid, and finding alternative routes consumes network resources. Multipath routing (Radi et al. 2012; Vaidya et al. 2008) appears to be a promising technique for ad hoc routing protocols to solve this problem. However, there has not been general analysis of the optimal utilization of multipath routing. For instance, the network power consumption may reduce if the source moving node obtains many paths to each destination by reducing the number of route discovery, however, that increases the number of route replies required to establish the routes which increases the network power consumption. Therefore, the number of paths is a significate factor that effect network performance and required to be careful consider.

Most of the proposed multipath routing protocols allow the destination node to select the paths based on the degree of disjointedness among paths found, however the necessity of this assumption is debatable (Rookhosh et al. 2008; Zafar et al. 2007) and does not consider the distribution of neighbour nodes around the source node. For instance, if a moving source node has multiple paths to a destination via neighbours located very close to each other, the links with these neighbours disconnected quickly when the source node moves in an opposite direction to these neighbours and hence the source loses the benefit of having alternative paths to its destination.

An on-demand multipath routing scheme is presented by Liu and Liu as a multipath extension of Dynamic Source Routing (DSR) (Liu and Liu 2010). They proposed the destination node selects disjoint paths with minimum end to end delay according to the accumulated delay calculated by the intermedin nodes. Omar et. at. (Omar et al. 2011) considered the residual energy of the nodes in the selection of paths. They proposed on-demand multipath protocol that the source node selects disjoint paths with the maximum residual energy. However, they did not consider the neighbour nodes and the number of paths. Tsirigos and Haas (Tsirigos and Haas 2004a) and (Tsirigos and Haas 2004b) considered load balancing by distributing data load over multiple paths. 
It was found that the probability of successful communication of packets increases with the number of used paths. However, the analysis does not consider the power consumption induced by acquiring multiple paths. Pham and Perreau (Pham and Perreau 2003) compared the overhead of reactive multipath routing with single path routing when all nodes in the network are mobile. They showed that the overhead increases significantly when the number of multiple paths exceeds three. However, this result did not consider the arrangement of neighbour nodes in the discovered routes.

The main contributions of this paper are the following: (i) We consider a multipath routing scheme, where the destination stores all the copies of routes which arrived, in order to determine which requests to respond to based on the distribution of the neighbours of the source node. (ii) We explore how the number and spread of neighbour nodes used by the source node to reach the destination in a multipath routing protocol affects the total network power consumption. (iii) proposing an analytical model to calculate the network power consumption when the source node moves in arbitrary directions and speeds. We assume each route starts with a different neighbour node that well distributed around the source node, as then a link failure with a neighbour in one route does not effect the others. Different criteria are suggested to determine the number of alternative routes used by the protocol (Nasipuri et al. 2001). We will later show that the best results are achieved with about six routes.

The remainder of this paper is organized as follows. In Section 2 we set up the mathematical framework for power consumption. In Section 3 we introduce the geometry of the source node and its neighbours. In Section 4 we develop expressions for expected power consumption for a multipath routing protocol. In Section 5 we present a comparison of theoretical and simulation results, with conclusions in Section 6.

\section{TOTAL POWER CONSUMPTION}

We will refer to total power consumption as the power consumed by the network nodes in order for the source node to obtain single/multiple routes, in addition to the power required to forward datapackets to a destination. In this research we are interested in minimizing total power consumption by selecting the number of multipath routes needed to send data from source to destination. Accordingly, we assume that all the nodes consume the same amount of power for sending a data-packet, $P_{d}$, and routing packets (route request, route reply) are transmitted at default power, $P_{r}$.
In multipath routing the source node obtains multiple routes to the destination using a single route discovery process. The source node, $n_{s}$, selects a valid route (of length $\left(h_{d}+1\right)$ ) from its cache, then $\left(h_{d}+1\right) P_{d}$ is the power consumed by sending a datapacket to $n_{d}$. If all the routes to $n_{d}$ are expired, a new route discovery process must by carried out. We assume that the route discovery process reveals a path through each of the $K$ neighbours, and that the number of hops from neighbour $i$ to $n_{d}$ is $h_{i}$. The power consumed for flooding the route request is $(n-1) P_{r}$, where $n$ is the total number of nodes in the network, plus the route replies along each of the $K$ paths. Let $t_{a}$ be the time of arrival of a new packet destined for $n_{d}$, and $T$ be the route expiry time for the largest surviving route. The total power consumed is given by

$$
\mathrm{PC}=\left\{\begin{array}{rr}
\left(h_{d}+1\right) P_{d} & t_{a}<T \\
(n-1) P_{r}+\Sigma_{i=1}^{K}\left(h_{i}+1\right) P_{r} & \\
+\left(h_{d}+1\right) P_{d} & t_{a}>T
\end{array}\right.
$$

In order to determine the time $T$ when routes break, we need to make reasonable assumptions about the arrangement and movement of the nodes. For simplification we assume that route breakages only occur due to source node movement. Our chosen network topology is discussed in the following section.

\section{TOPOLOGY SCENARIO}

We assume that each node is equipped with an omnidirectional antenna and that signal attenuation is due only to path loss related to distance transmitted. We assume that the transmission ranges of all nodes are identical and equal to $r$. We assume that the velocities of the neighbour nodes are slow enough to be considered stationary and that $n_{s}$ moves with a constant speed, $v$, in a random direction, $\theta_{s}$. The scenario could also be extended such that the speeds of the neighbouring nodes are combined with the speed of $n_{s}$, and $v$ is the relative speed. This scenario fits well with the random waypoint mobility model. All node-to-node communications are assumed to be bi-directional.

We assume that the source node has a set of well distributed neighbours. This can be achieved in a dense network using, for example, topology control, where a set of neighbour nodes is selected by dividing a disk centred at the source node into a number of cones by lines. The angle of the maximum cone is no more than $\alpha$ and one neighbour node is selected for every cone (Wu and Dai 2006; Poduri et al. 2009). 


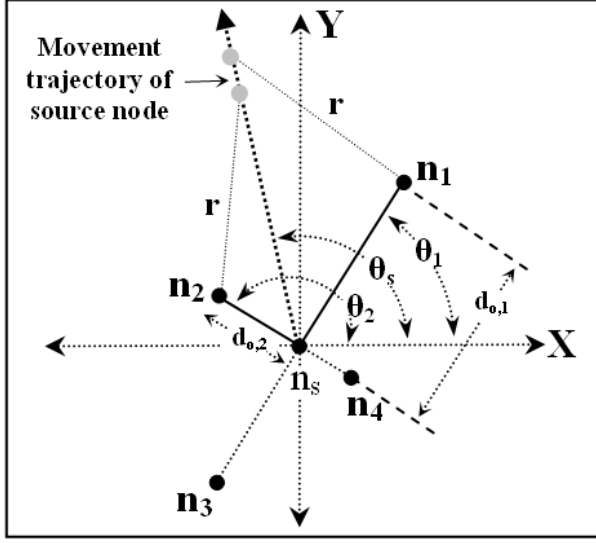

Figure 1: An example of four neighbour nodes at equal angles and varying distances spread around the source node, $n_{s} . n_{1}$ and $n_{2}$ are adjacent neighbours.

\subsection{Link Residual Time}

The time from when $n_{s}$ moves from its initial position to the point where the link with $n_{i}$ breaks is called the link residual time, $R_{i}$. The link residual time is shortest if the source node moves exactly between two neighbours. We assume this worst case scenario as illustrated in Fig 1. To determine when the link between the source node and the nearest neighbour breaks, we let the angle between $n_{s}$ and its neighbours be $\left|\theta_{s}-\theta_{i}\right|=\left|\theta_{s}-\theta_{i+1}\right|=\pi / K$, where $\theta_{s}$ is the direction of movement of $n_{s}$, and $\theta_{i}$ and $\theta_{i+1}$ are angles of adjacent neighbour nodes. The distance that $n_{s}$ has to travel in direction $\theta_{s}$, before the link with its adjacent neighbours breaks is given by

$$
d_{i}=d_{o, i} \cos \left(\theta_{s}-\theta_{i}\right)+\sqrt{r^{2}-d_{o, i}^{2} \sin ^{2}\left(\theta_{s}-\theta_{i}\right)}
$$

If $n_{s}$ moves distance $d_{i}$ at velocity $v$ then, $R_{i}=d_{i} / v$. Assume $d_{o, i} \sim U[0, r)$, then the PDF and CDF of the link residual time for different values of $K$ are as follows:

\subsubsection{Two Neighbours}

The link residual time, is equal to $r / v$ and 0 respectively when $d_{o, i}$ is at its limiting values $0, r$. The PDF and CDF of the link residual time is given by

$$
\begin{gathered}
f_{R_{i}}\left(r_{i}\right)=\frac{r_{i} v^{2}}{r \sqrt{r^{2}-r_{i}^{2} v^{2}}} \\
F_{R_{i}}\left(r_{i}\right)=1-\frac{\sqrt{r^{2}-r_{i}^{2} v^{2}}}{r}
\end{gathered}
$$

\subsubsection{Three Neighbours}

The link residual time, is equal to $r / v$ for $d_{o, i}$ equal to both 0 and $r$. The maximum link residual time $R_{i}=$ $r /(v \sin (\pi / 3))$ is achieved when $d_{o, i}=r / \tan (\pi / 3)$. Then the PDF and CDF of the link residual time is given by

$$
\begin{gathered}
f_{R_{i}}\left(r_{i}\right)=\frac{2 v^{2} r_{i} \sin ^{2}(\pi / K)}{r \sqrt{r^{2}-v^{2} r_{i}^{2} \sin ^{2}(\pi / K)}} \\
F_{R_{i}}\left(r_{i}\right)=1-\frac{2}{r} \sqrt{r^{2}-v^{2} r_{i}^{2} \sin ^{2}(\pi / K)}
\end{gathered}
$$

\subsubsection{Four or More Neighbours}

The link residual time, is equal to $r / v$ for $d_{o, i}=0$. The maximum link residual time $R_{i}=2 r \cos (\pi / K) / v$ is achieved when $d_{o, i}=r$. The PDF and CDF of the link residual time is given by

$$
\begin{aligned}
& f_{R_{i}}\left(r_{i}\right)=\frac{1}{r}\left(v \cos (\pi / K)+\frac{v^{2} r_{i} \sin ^{2}(\pi / K)}{\sqrt{r^{2}-v^{2} r_{i}^{2} \sin ^{2}(\pi / K)}}\right) \\
& F_{R_{i}}\left(r_{i}\right)=\frac{1}{r}\left(v r_{i} \cos (\pi / K)-\sqrt{r^{2}-v^{2} r_{i}^{2} \sin ^{2}(\pi / K)}\right)
\end{aligned}
$$

\subsection{Packet Arrival Time}

Packet arrival times, $t_{a}$, are generally modelled as having an exponential distribution, with parameter $\lambda_{a}$ appropriate to the given network. We use this model here. The PDF and CDF of arrival times is given by

$$
\begin{gathered}
f_{a}(t)=\lambda_{a} e^{-\lambda_{a} t} \\
F_{a}(t)=1-e^{-\lambda_{a} t}
\end{gathered}
$$

In this section we have presented a statistical model of the topology scenario. We will use this model to develop an analytical model of the expected power consumption.

\section{EXPECTED POWER CONSUMPTION}

We consider the expected power consumed with the occurrence of data-packets requests and new route requests. The expected value of the power consumption is equal to the cost of an individual data-packet request, multiplied by the probability that there is an unexpired route in the cache, in addition to the cost of an individual route discovery process, multiplied by the probability that the route is necessary. The probability of route discovery is determined by the topology scenario and the caching strategy.

Assume the source node $n_{s}$ and destination $n_{d}$ are initially separated by distance $L_{d}$. The path length 
is longest if the neighbour node that is selected to forward data is located in the opposite direction to the destination, thus $\left|\theta_{d}-\theta_{i}\right|=\pi$, as illustrated in Fig. 2. Assume $d_{o, i} \sim U[0, r)$, and similarly for all hop lengths in each path then the expected length of each hop is $0.5 r$, then the expected distance between the sending neighbour node and destination is $L_{d}+0.5 r$, and the expected number of hops from the neighbour to the destination is $h_{d}=L_{d} / 0.5 r+1$.

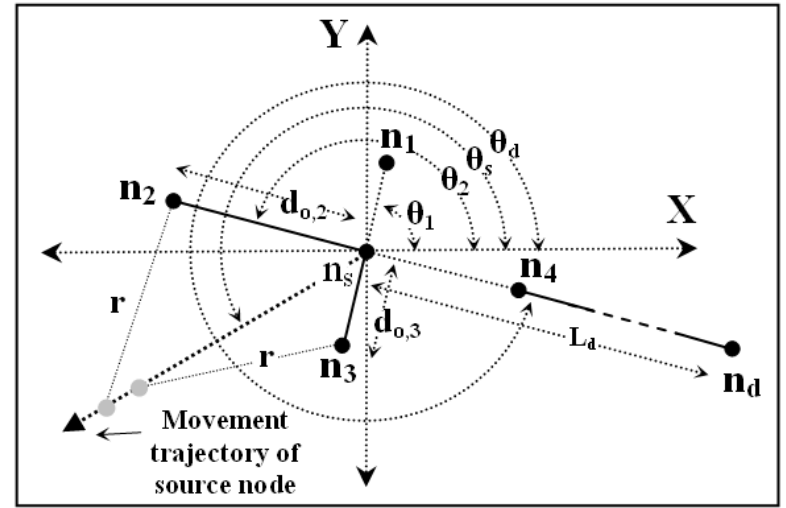

Figure 2: An example of a worst-case path length scenario illustrates the distance relationship between moving source node, $n_{s}$, active neighbour nodes, $n_{1}, n_{2}$, $n_{3}$ and $n_{4}$, and destination node, $n_{d}$.

The PDF of the maximum link residual time, $R_{\max }$, of the two closest neighbours is determined by finding the CDF first, as follows

$$
\begin{aligned}
F_{R_{\max }}(t) & =P\left\{R_{\max }<t\right\} \\
& =P\left\{\left(R_{i_{1}}<t\right) \cup\left(R_{i_{2}}<t\right)\right\} \\
& =F_{R_{i}}^{2}(t)
\end{aligned}
$$

Taking the derivative of (11) it can be seen that the PDF of the maximum $R_{i}$ value is given by

$$
f_{R_{\max }}(t)=2 F_{R_{i}}(t) f_{R_{i}}(t)
$$

Combining (1) and (12), and using the PDF and CDF obtained in Section 3.1, we can determine an expression for the expected power consumption, where

$$
\begin{aligned}
& E\{P C \mid K \text { paths }\} \\
& =\int_{R_{i, \min }}^{R_{i, \max }} E\left\{P C \mid R_{1}, R_{2}\right\} f_{R_{\max 2}}(t) d t \\
& =2\left(h_{d}+1\right) P_{d} \int_{R_{i, \min }}^{R_{i, \max }}\left(1-e^{-\lambda_{a} t}\right) F_{R_{i}}(t) f_{R_{i}}(t) d t \\
& +2\left((n-1) P_{r}+\left(h_{d}+1\right) P_{r}+\left(h_{d}+1\right) P_{d}\right) \\
& \cdot \int_{R_{i, \min }}^{R_{i, \max }} e^{-\lambda_{a} t} F_{R_{i}}(t) f_{R_{i}}(t) d t
\end{aligned}
$$

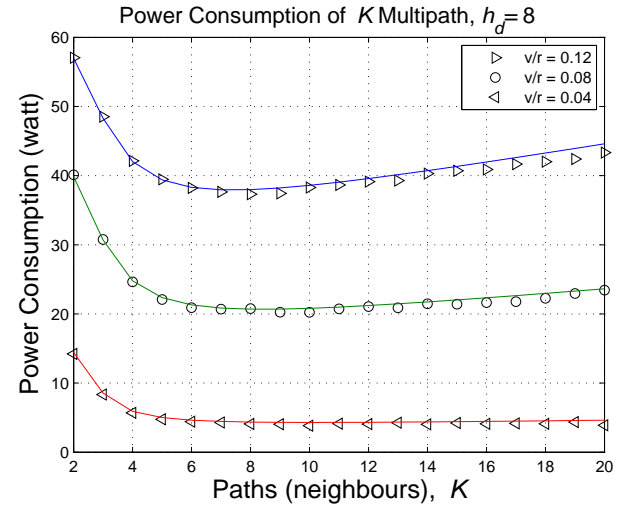

Figure 3: Network power consumption as it varies with source node velocity, from (13). Simulation results are depicted by markers while theoretical results are depicted by lines.

and the values of $R_{i, \min }$ and $R_{i, \max }$ depend on the number of neighbour nodes, $K$. There is no closed form solution to (13), so it must be calculated numerically.

\section{RESULTS}

In this section we present and discuss results of theoretical calculations and Monte-Carlo simulations, conducted in Matlab, for expected total power consumption, discussed above.

We assume a network with $n=400$ nodes, each node in the network has a default power level, $P_{r}=$ $280 \mathrm{~mW}$, for the transmission range, $r=250 \mathrm{~m}$. The node uses $P_{d}=140 \mathrm{~mW}$ for forwarding of datapacket to the destination. The source node sends data-packets to the destination at arrival time $t_{a}$ seconds while it moves in a random direction. It is assumed that the source node moves at low, medium and high speed with 10,20 and $30 \mathrm{~m} / \mathrm{s}$, respectively.

For each instance of the simulation, the source node direction $\theta_{s} \sim U[0,2 \pi)$, and the neighbour node location, $d_{o, i} \sim U[0, r)$ and $\theta_{i}$, are generated according to the scenario in Fig. 1. The source node generates packets according to an exponential distribution with parameter $\lambda_{a}=0.1$. The packet arrival time is compared to the link residual times, and the total power consumption is counted.

The expected power consumption for fixed path length $h_{d}$ is shown in Fig. 3. The results show that the number of paths, $K$, has a significant effect on power consumption. At low numbers of paths the power-consumption curves decrease with $K$, since an increase in the number of paths decreases the number of route discoveries by providing alternative routes to the destination. However, after a certain number of paths, the reduction in power 


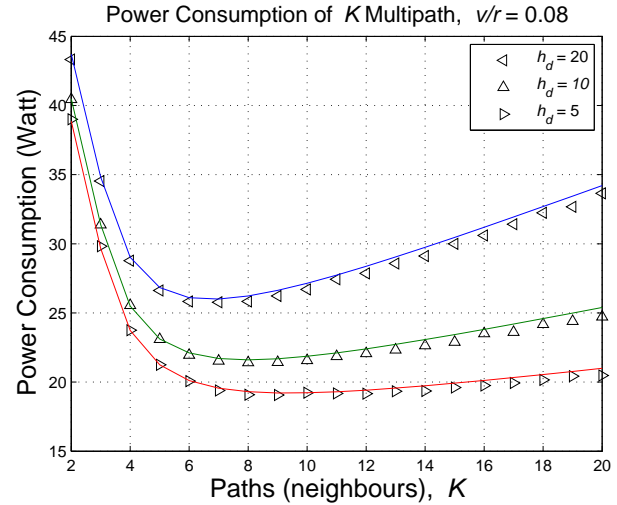

Figure 4: Network power consumption as it varies with $h_{d}$, from (13). Simulation results are depicted by markers while theoretical results are depicted by lines.

consumption due to providing alternative paths is outweighed by the power consumed by route replies for each discovered path. Therefore, increases to the number of paths past that point increases power consumption. We see from the results that the minimum network power consumption can be achieved when the source node has six multipath routes via neighbour nodes that are equally angled around the source node.

Fig. 4 shows the effect of the number of hops between the source and destination on the network power consumption at moderate speed of source node. The results show that power consumption increases with the number of hops, since that increases the number of nodes that participate in communication between the source and the destination. The result also shows that selecting six paths via neighbour nodes equally spread around the source nodes minimizes the network power consumption for different numbers of hops between the source and the destination, unless the destination is close, in which case up to eight paths may be useful.

\section{CONCLUSION}

In this paper the number of paths in a multipath routing protocol and the distribution of neighbour nodes in the discovered routes are studied when the source node moves in arbitrary directions and speeds. We assume that the protocol identifies separate routes through all active neighbours of the source node. An analytical model is developed and verified by simulation. The results show that the total network power consumption is dependent on the number of paths corresponding to the neighbours of the source node. If the number of paths is too low, the power consumption is increased because of increased need for route discovery, but if the number of paths is high, the network power consumption is increased because of an increased number of route reply packets. This becomes more pronounced with increasing mobility and greater source-destination separation. We show that, in most cases, the total network power consumption is minimized when the moving source node has six well distributed neighbour nodes.

\section{REFERENCES}

Shun Liu and Jian Liu. Delay-aware multipath source routing protocol to providing QoS support for wireless ad hoc networks. In Proc. of Communication Technology Conference, IEEE, pages 1340-1343, 2010.

Asis Nasipuri, Robert Castaneda, and Samir R. Das. Performance of multipath routing for on-demand protocols in mobile ad hoc networks. Mobile Networks and Applications, 6(4):339-349, August 2001.

Smail Omar, Mekkakia Zoulikha, and Bernard Cousin. Energy efficiency in ad hoc wireless networks with node-disjoint path routing. In Proc. of Systems, Signal Processing and their Applications Conference, IEEE, pages 127-130, 2011.

Peter P. Pham and Sylvie Perreau. Performance analysis of reactive shortest path and multi-path routing mechanism with load balance. In Proc. of INFOCOM 2003 Conference, IEEE, volume 1, pages 251-259, 2003.

Sameera Poduri, Sundeep Pattem, Bhaskar Krishnamachari, and Gaurav S. Sukhatme. Using local geometry for tunable topology control in sensor networks. IEEE Transactions on Mobile Computing, 8(2):218-230, February 2009.

Marjan Radi, Behnam Dezfouli, Kamalrulnizam Abu Bakar, and Malrey Lee. Multipath routing in wireless sensor networks: Survey and research challenges. Sensors, (12):650-685, 2012.

Fahimeh Rookhosh, Abolfazl Toroghi Haghighat, and Saeed Nickmanesh. Disjoint categories in low delay and on-demand multipath dynamic source routing adhoc networks. In Proc. of Distributed Framework and Applications Conference, pages 207-213, 2008.

Aristotelis Tsirigos and Zygmunt J. Haas. Analysis of multipath routing-part I: the effect on the packet delivery ratio. IEEE Transactions on Wireless Communications, 3(1):138- 146, January 2004a.

Aristotelis Tsirigos and Zygmunt J. Haas. Analysis of multipath routing, part 2: mitigation of the effects 
of frequently changing network topologies. IEEE Transactions on Wireless Communications, 3(2): 500-511, March 2004b.

Binod Vaidya, Dong-You Choi, Jong-An Park, and Seung Jo Han. Multipath routing scheme for wireless multihop network. In Proc. of ICCSA (2) Conference, pages 433-445, 2008.

Jie Wu and Fei Dai. Mobility-sensitive topology control in mobile ad hoc networks. IEEE Transactions on Parallel and Distributed Systems, 17(6):522-535, June 2006.

Haseeb Zafar, David Harle, Ivan Andonovic, and Mahmood Ashraf. Partial-disjoint multipath routing for wireless ad-hoc networks. In Proc. of Local Computer Networks (LCN 2007) Conference, IEEE, pages 258-259, 2007. 